\title{
P67 is involved in the regulation of cytoskeleton dynamics by modulating the expression and activity of Pak1 in differentiated C2C12 myoblasts
}

\author{
Bansidhar Datta* and Rekha Datta \\ Department of Chemistry and Biochemistry, \\ Kent State University, Kent, $\mathrm{OH}$ \\ bdatta@kent.edu
}

\begin{abstract}
Differentiation of skeletal myogenic cells into multinucleated myotubes involves several cellular events including survival, migration, and motility. The level of eukaryotic initiation factor 2 (eIF2)-associated glycoprotein 667 gradually increases during the differentiation of C2C12 myoblasts into myotubes. It not only suppresses the rates of global protein synthesis, it inhibits the activation and activity of extracellular signalregulated kinases 1 and 2 (ERK1/2) when myoblasts are differentiating into myotubes. To explore whether p67 has additional roles in myoblasts' survival, migration, and motility we examined p67's effects on expression and activity of p21-activating kinase 1 (Pakl). Because Pak1 not only controls several cellular activities including glucose homeostasis, it is also involved in cytoskeleton dynamics by regulating cell migration and motility. We found that Pakl level is increased by $\sim 1.5$ fold in rat p67-expressing myoblasts and its conserved D251 and $H 331$ residues along with its $N$-terminal acidic residue-rich domain are involved in this process. On the other hand, the level of Pakl is 3.0 -fold higher in rat p67-expressing myotubes and its $N$-terminal acidic residuerich domain but not the lysine-rich domains 1 \& II is involved in this process. P67 is also involved in the increased level of $\mathrm{Cdc42}$ by proteolytic processing of the "pro" form of this protein, which is the upstream activator of Paks. Together, these results suggest that p67 is involved in the upregulated expression and activity of Pakl in C2C12 myotubes and thus may be involved in the control of cytoskeleton dynamics including survival, migration, and motility of differentiated C2C12 myoblasts.
\end{abstract}

Abbreviations used: p67, a $67 \mathrm{kDa}$ glycoprotein that binds to both eukaryotic initiation factor 2 (eIF2) and extracellular signal-regulated kinases 1 \& 2 (ERK1/2); D251A, a p67 point mutant, which has alanine substitution for aspartic acid at 251 amino acid residue; D6/2, a p67 block mutant, where a stretch of $N$ terminal acidic amino acid residues has been replaced with uncharged amino acids; K1K2, a p67 block mutant, where both of its $N$-terminal lysine/arginine-rich stretches of amino acid residues have been replaced with uncharged amino acids; D6/2+D251A, a D6/2 mutant containing a second site point mutation, where its 251 aspartic amino acid residue has been changed to alanine; D6/2+H331A, a D6/2 mutant containing a second site point mutation, where its histidine amino acid residue at position 331 has been changed to alanine; Pakl, the p21-activating kinase 1 and $V$, vector expressing enhanced green fluorescence protein (EGFP).

Keywords: eIF2-associated glycoprotein, p67; Pak1, C2C12 myoblasts and myotubes, p67 mutants

\section{INTRODUCTION}

Skeletal muscle differentiation is one of the most important cellular events. During myogenesis, myogenic progenitor cells differentiate, migrate, and fuse with one another to generate multinucleated myotubes and later myofibers (1). $\mathrm{C} 2 \mathrm{C} 12$ mouse myoblasts has been used as an excellent model system to study the detailed molecular mechanism of fusion of myoblasts into multinucleated myotubes (2). A series of events for example, (i) suppression of growth promoting signals including protein synthesis (3-5), (ii) activation of metalloproteinases (6), (iii) cleavage of MARCKS and other substrates (7-8), (iv) migration of myoblasts (9), (v) survival and alignment of myoblasts (10), (vi) dynamic changes of plasma membranes of myogenic cells such as inward membrane invaginations occurring with endocytosis and outward membrane protrusions during migration of myoblasts (11), and finally (vii) fusion of myoblasts into myotubes (12), takes place while myoblasts are fusing into myotubes to make myofibers. Several molecules starting from cell cycle control proteins, myogenic factors, growth regulatory proteins, translation regulatory protein, and proteinases play important roles during these processes (12). 
The level of eukaryotic initiation factor 2 (eIF2)-associated glycoprotein, p67 gradually increases while myoblasts are differentiating into myotubes $(3,13)$. During this period, p67 dissociates from eIF2 and this dissociation causes higher levels of eIF2 $\alpha$ phosphorylation that suppresses the rates of global protein synthesis (5). At the same time of differentiation, p67 associates with extracellular signal-regulated kinases 1 and 2 (ERK1/2) and inhibits their phosphorylation. This later event ultimately leads to the down regulation of ERK1/2 activation and activity $(3-4,13)$, which is essential for permanent with draw of myoblasts from cell cycle while fusing into myotubes $(1,12)$. Rat p67, which is 480 amino acids long, has two segments - the N-terminal 1-107 amino acid residues or p26 segment and downstream 108-480 amino acid residues or p52 segment. At the p26 segment, there are two lysine residue-rich domains I \& II separated by an acidic residue-rich domain (13-14). Next to the lysine residue-rich domain $\mathrm{I}$, there is a O-glycosylation motif and together they regulate the phosphorylation of eIF $2 \alpha$ by binding to the lysine residue-rich domain II and 340-430 amino acids region through eIF2 $\gamma$-subunit (15-17). The downstream 211- 430 amino acids region of p67 also binds to ERK1/2 MAP kinases and regulates their activation and activity by inhibiting ERK1/2 phosphorylation $(4,18)$. At the p52 segment, there are five conserved amino acid residues - D251, D262, H331, E364, and E459, which upon folding creates a shallow groove for the substrate-binding pocket, where either p26 segment or other proteins can bind to and get close to p67's catalytic site H231 residue $(13,19)$. This site is used either for auto-proteolysis or intermolecular proteolysis reactions (19-20). EIF2 $\gamma$-subunit or ERK1/2 MAP kinases when bind to p67, they cover both the catalytic site and the substrate-binding pocket and inhibit p67's proteolytic activity $(13,16,18)$. At the same time, p67's N-terminal lysine residue-rich domain I and O-glycosylation domain act cooperatively to inhibit the phosphorylation of eIF2 $\alpha$ or ERK1/2 MAP kinases $(13,15)$. Apart from p67's roles in the suppression of growth promoting signals leading to differentiating myoblasts, we wondered whether p67 has any role(s) during myoblasts' migration and fusion by modulating the cytoskeleton. A family of p21-activating kinases (Paks) is involved in controlling cytoskeleton dynamics (21). There are two groups of Pak - Pak 1, 2, and 3 belong to group A and Pak 4, 5, and 6 belong to group B. Pak1 is found in several tissues including mammary gland, muscle, and spleen $(22-23)$.

In this study, we examined whether p67 has any role(s) in the expression and activity of Pak1 in $\mathrm{C} 2 \mathrm{C} 12$ myoblasts and myotubes. We found that rat p67 increased Pak1 level by more than $50 \%$ in $\mathrm{C} 2 \mathrm{C} 12$ myoblasts and its lysine residue-rich domains I \& II are not involved in this increment whereas, its acidic residue-rich domain and D251 residue are essential for this activity. This later domain and the aspartic amino acid residue at position 251 and histidine residue at position 331 act cooperatively. We also noticed that rat p67 increased Pak1 level by more than 3 -fold in $\mathrm{C} 2 \mathrm{C} 12$ myotubes and its aspartic amino acid residue at position 251 and the $\mathrm{N}$-terminal lysine residue-rich sequences are not involved in this process. In contrast, p67's acidic residue-rich sequences play important role(s) in this process. Altogether, our data suggest that p67 is involved in the regulation of cytoskeleton dynamics including survival, migration, and motility of differentiated $\mathrm{C} 2 \mathrm{C} 12$ myoblasts by increasing the expression and activity of Pak1.

\section{Materials AND Methods}

All chemicals used in this study were obtained from Sigma Chemicals (St. Louis, MO), Merck (Darmstadt, Germany), ICN Biomedicals, Inc. (Aurora, Ohio), Fisher Chemicals (New Jersey), or GIBCO-BRL (Rockville, MD). All enzymes used in this study were purchased from New England Biolabs (Beverly, MA). Molecular mass markers were purchased from BioRad.

\subsection{Antibodies}

Monoclonal antibodies specific to Pak1 (Sc-881) and Cdc42 (Sc-8401) were purchased from Santa Cruz Biotechnology. A monoclonal antibody specific to $\alpha$ Actin was purchased from Sigma (Sigma Biochemicals, St. Louis, MO). Tagged secondary antibodies for Western blots were obtained with the ECL kit (Perkin Elmer).

\subsection{Site-Directed Mutagenesis and Subcloning into Mammalian Expression Vector}

A $~ 1.4 \mathrm{~kb}$ cDNA insert encoding the entire p67 coding region was produced by PCR using appropriate forward and reverse primers (5' TCCCCCGGGTGATGGCGGGCGTGGAAGAG 3' and 5' TCCCCCGGGAAGTTTTAATAGTCATCTCCTC 3' respectively) with the pGEM-p67 template 
P67 is involved in the regulation of cytoskeleton dynamics by modulating the expression and activity of Pak1 in differentiated C2C12 myoblasts

(24). The resulting DNA fragment was gel purified, digested with $S m a \mathrm{I}$, and ligated in M13mp18 (Stratagene) at the SmaI site. A single-stranded uracil template was made, and annealing reactions were performed with mutant oligo nucleotides. A detailed description for the generation of specific p67 mutants and their sequence variations as compared to wild type rat p67 was reported (25). The cDNA inserts for p67 with mutations at specific domains or sites were isolated from the RF form of M13mp18, digested with XmaI, and the DNA inserts were ligated at the XmaI site of pEGFP-C3 vector (Clontech, Palo Alto, CA). Plasmids with sense orientation were selected for further analysis. The specific fusion junction between mutant cDNA of p67 and enhanced green fluorescent protein (EGFP) was confirmed by DNA sequencing. All procedures for manipulation of recombinant DNA were either published earlier $(4,25-27)$ or followed from molecular cloning, a laboratory manual (28), and current protocols in molecular biology (29).

\subsection{Cell Culture and Generation of Stable Cell Lines}

$\mathrm{C} 2 \mathrm{C} 12$ mouse myoblasts (from American Type Culture Collection) cultures were maintained in growth medium as described $(3-5,30)$. 50-60\% confluent cultures of $\mathrm{C} 2 \mathrm{C} 12$ cells were transfected with plasmids complexed with Superfect following protocols described by the manufacturer (Qiagen). Cells constitutively expressing either EGFP or EGFP-fusions of p67 or its specific mutants were selected by treating cells with G418 following the procedures as described $(4,30)$. C2C12 myoblasts constitutively expressing EGFP, or its various in-frame fusions of rat p67 and its specific mutants were grown in growth medium to confluence and then allowed to differentiate into myotubes for $96 \mathrm{~h}$ or $120 \mathrm{~h}$ in differentiation medium following the procedures as described (3-5, 30). Culturing conditions and generations of stable rat tumor hepatoma (KRC-7) cells were the same as described (26-27).

\subsection{Cell Lysate Preparation and Western Blot Analysis}

Procedures for preparation of cell lysates either from myoblasts or myotubes and Western blots were essentially the same as described $(3-5,30)$. Protein bands in Western blots were scanned and their intensities were quantitatively measured by NIH Image 162 software program.

\section{RESUlts}

P67 level gradually increases during differentiation of $\mathrm{C} 2 \mathrm{C} 12$ myoblasts into myotubes (3-5). To examine whether this increased p67 level has any influence in the expression of p21-activating kinase 1 (Pak1), we generated stable C2C12 cell lines constitutively expressing EGFP and EGFP-fusions of rat p67 and its mutants, D251A, D6/2, K1K2, and double mutants D6/2+D251A and D6/2+H331A. These myoblasts cell lines were grown in growth medium containing G418 and cell extracts were prepared. The levels of Pak1 and $\alpha$ Actin were measured in these cell extracts by Western blots using monoclonal antibodies specific to these proteins (Fig. 1). We found more that 50\% increase of Pak1 in C2C12 myoblasts constitutively expressing wild type rat p67 and its K1K2 mutant (Fig. 1A, lanes 2 and 5) whereas, p67's other mutants D251A and D6/2 inhibited this increment by more than $50 \%$ as compared to control cells expressing empty vector (Fig. 1A compare lanes 3 and 4 with lane 1). Although, D6/2 mutant inhibited the Pak1 expression, its second site mutants D6/2+D251A and D6/2+H331A reversed this inhibition (Fig. 1A, lanes 6 and 7). These results suggest that p67 is involved in the increased expression of Pak1 in $\mathrm{C} 2 \mathrm{C} 12$ myoblasts and its $\mathrm{N}$-terminal acidic residuerich sequences and D251 residue play important role(s) in this process whereas, its $\mathrm{N}$-terminal lysine residue-rich sequences are not involved.

To examine whether p67 has any effect on expression of Pak1 while the above cell lines were grown in differentiation medium, we performed similar analyses for the levels of Pak1 and aActin as a control in total protein samples from $\mathrm{C} 2 \mathrm{C} 12$ myotubes constitutively expressing rat p67 and its certain mutants, which are mentioned earlier and the results are shown in Figure 2. Our results show more than 3-fold increase of Pak1 level in $\mathrm{C} 2 \mathrm{C} 12$ myotubes constitutively expressing rat p67 and its mutants D251A and $\mathrm{K} 1 \mathrm{~K} 2$ as compared to control myotubes constitutively expressing the empty vector (Fig. 2A, compare lanes 2, 3, and 5 with lane 1). In contrast, this increment of Pak1 level was decreased to less than 2-fold in $\mathrm{C} 2 \mathrm{C} 12$ myotubes constitutively expressing p67 mutants like D6/2 block mutant (Fig. 2A, lane 4) and its double mutants D6/2+D251A and D6/2+H331A (Fig. 2A, lanes 6 and 7). These results thus suggest that p67 is involved in increased expression of Pak1 in $\mathrm{C} 2 \mathrm{C} 12$ myotubes and its $\mathrm{N}$-terminal acidic residue-rich sequences play an important role in this process. 


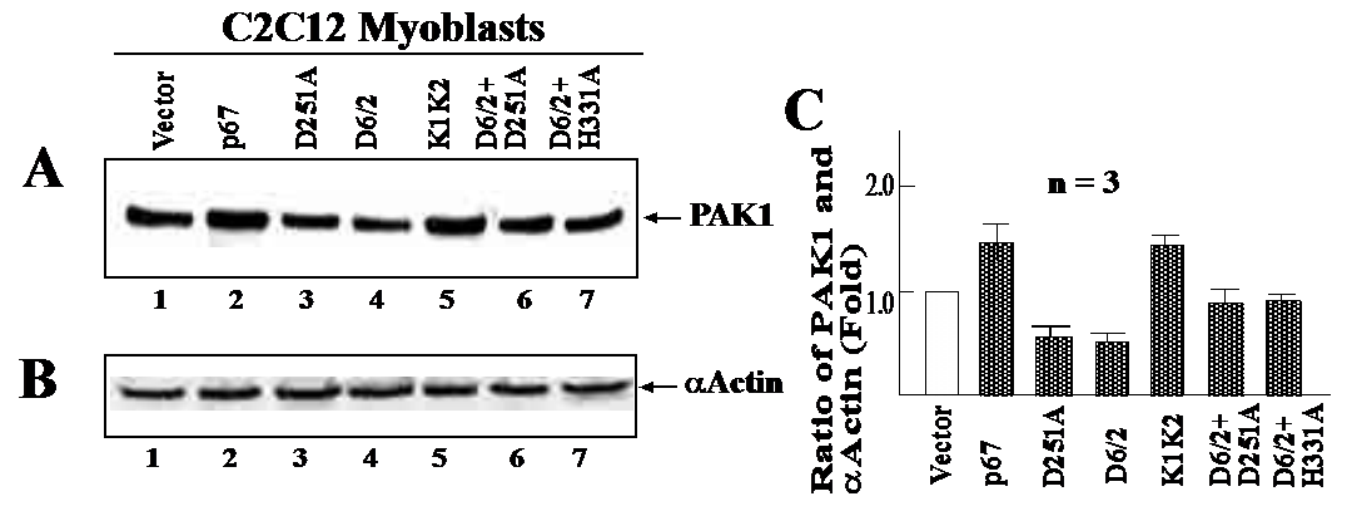

Fig1. Levels of Pak1 in C2C12 myoblasts constitutively expressing rat p67 and its mutants D251A, D6/2, $\mathrm{K} 1 \mathrm{~K} 2, \mathrm{D6} / 2+\mathrm{D} 251 \mathrm{~A}$, and D6/2+H331A. Stable cell lines constitutively expressing EGFP expresion vector, and its in-frame fusions of rat p67 or its various mutants were generated. Cell lysates from these cell lines were made and analyzed for the levels of Pakl (A) and aActin (B) on Western blots using antibodies specific to these proteins. After scanning the band intensities from panel $A$ and panel $B$, the ratios of Pakl and $\alpha$ Actin were calculated and plotted on a graph $(C)$. The results are from three independent experiments $(n=3)$.

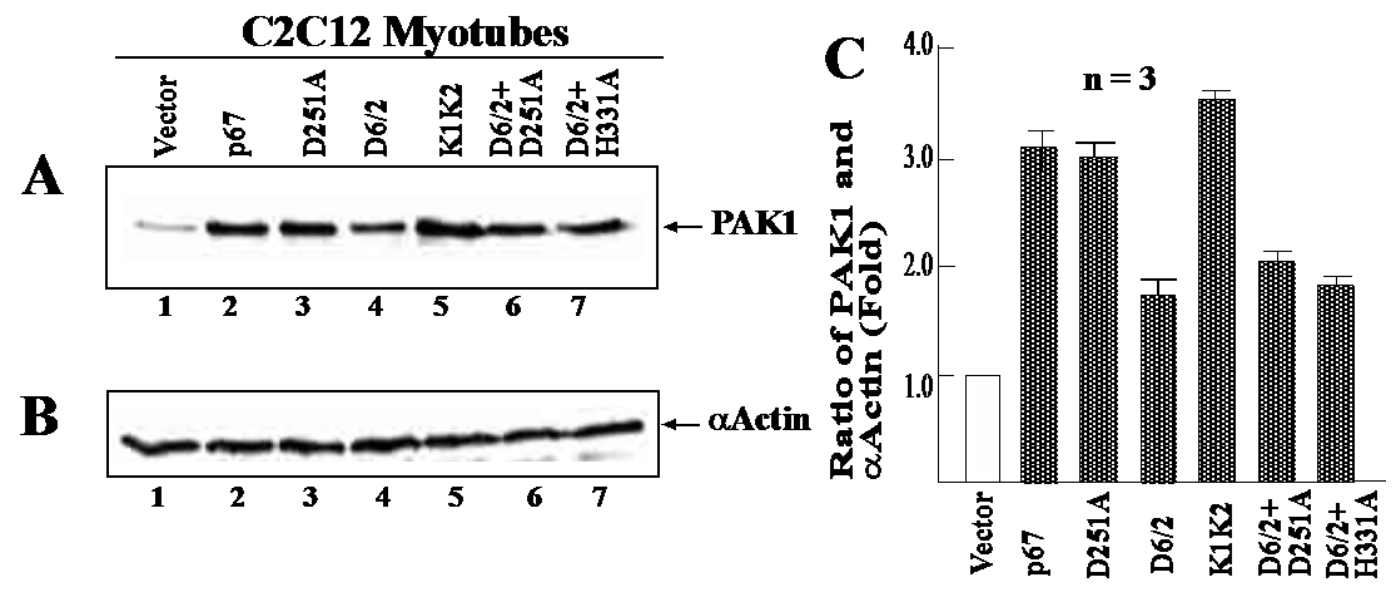

Fig2. Levels of Pak1 in C2C12 myotubes constitutively expressing rat p67 and its mutants D251A, D6/2, $\mathrm{K} 1 \mathrm{~K} 2, \mathrm{D6} / 2+\mathrm{D251A}$, and D6/2+H331A. C2C12 myoblasts constitutively expressing EGFP vector or EGFPfusions of p67, D251A, D6/2, K1K2, D6/2+D251A, and D6/2+H331A mutants were allowed to differentiate into mytubes in differentiation medium for $120 \mathrm{~h}$. Lysates were prepared after harvesting myotubes and analyzed for the levels of Pakl (A) and aActin (B) on Western blots using antibodies specific to these proteins. After scanning the band intensities from panel $A$ and panel $B$, the ratios of Pakl and $\alpha$ Actin were calculated and plotted on a graph $(C)$. The results are from three independent experiments $(n=3)$.

Next, we examined the level of Pak1 and its upstream activator Cdc42 Rho-GTPase in un-transfected $\mathrm{C} 2 \mathrm{C} 12$ myoblasts undergoing differentiation into myotubes. For this purpose, growth medium from confluent $\mathrm{C} 2 \mathrm{C} 12$ myoblast cultures were replaced with differentiation medium and allowed to differentiate for $120 \mathrm{~h}$. Total protein samples were prepared from different time intervals and analyzed for the levels of Pak1 (Fig. 3A) and Cdc42 (Fig. 4A). For comparison, the levels of $\alpha$ Actin were also measured in both cases (Figs. 3B and 4B). Our results show very slight variation of the levels of these two proteins while $\mathrm{C} 2 \mathrm{C} 12$ myoblasts were differentiating into myotubes. Since we found no significant change of Pak1 level and the level of its upstream activator Cdc42 during normal differentiation of $\mathrm{C} 2 \mathrm{C} 12$ myoblasts, we then asked whether overexpression of p67 or some of its mutants make any difference in Cdc42 level. For this purpose, we generated KRC7 cells constitutively expressing rat p67 and its mutants D6/2, K1K2, D251A, and D262A. Cell extracts were prepared from these cell lines and total protein samples were analyzed for the level of Cdc42 in Western blots using monoclonal antibodies specific to Cdc42 (Figs. 5A and 5C) and $\alpha$ Actin (Figs. 5B and 5D). We detected low level of Cdc42 in cells expressing EGFP alone (Fig. 5A, lane 1) and this level increased to 2.5-3 fold in rat p67-expressing KRC-7 cells (Fig. 5A, lane 2). In addition to the detection of full-length $\mathrm{Cdc} 42$, we also detected a slower migrating Cdc42 ("pro" form of Cdc42) more prominently in p67-expressing cells (Fig. 5A \& C, lanes 2). This form of Cdc42 however disappeared in both D6/2 and K1K2-expressing cells and full-length Cdc42 level increased to >5-fold as compared to p67-expressing cells (compare lanes 3-4 with lane 2 in Fig. 5A). Constitutive 
P67 is involved in the regulation of cytoskeleton dynamics by modulating the expression and activity of Pak1 in differentiated C2C12 myoblasts

expression of either D251A or D262A mutant showed no processing of the slower migrating "pro" form of $\mathrm{Cdc} 42$ to its mature form and there was a decrease in these forms of Cdc 42 in general (Fig. 5C, lanes 3-4). Taken together, these data indicate that p67 is involved in the increased levels of $\mathrm{Cdc} 42$ and its $\mathrm{N}$-terminal acidic residue-rich domain and lysine residue-rich domain I \& II are inhibitory to this maturation of Cdc42 from its "pro" form. On the other hand, p67's conserved D251 and D262 residues are not involved in this maturation process.

\section{A Differentiation of $\mathrm{C2C12}$ myoblasts}

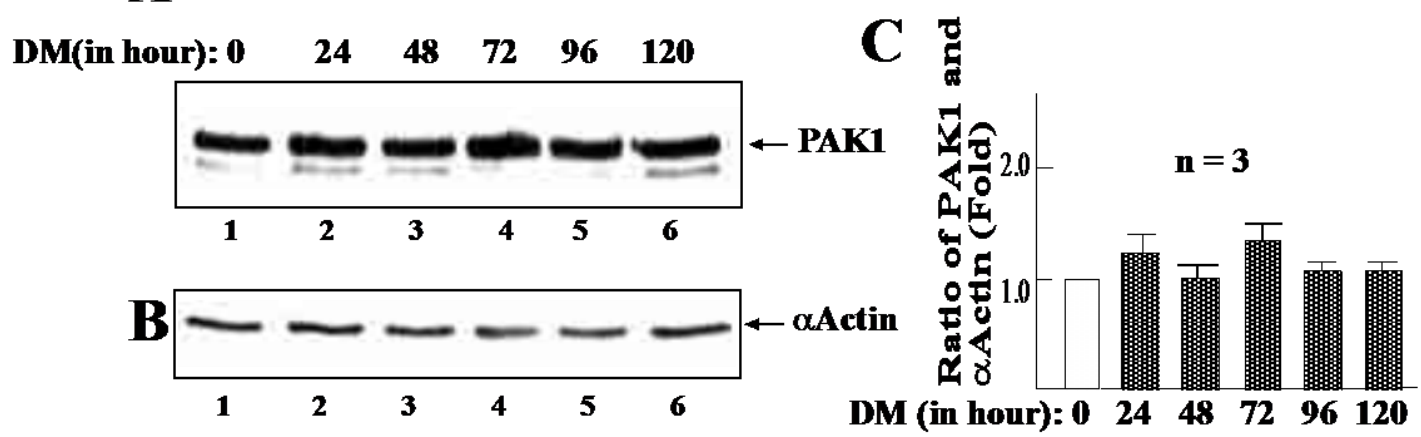

Fig3. Levels of Pak1 in C2C12 mouse myoblasts undergoing differentiation into myotubes. C2C12 myoblasts were grown to confluency and then allowed to grow in differentiation medium for five days. Cells were harvested and lysates were prepared every $24 \mathrm{~h}$ intervals and stored frozen at $-80^{\circ} \mathrm{C}$. At the end of differentiation periods total proteins from different time intervals were analyzed on Western blots for the levels of Pakl (A) and aActin (B) using specific antibodies to these proteins. After scanning the band intensities from panel $A$ and panel $B$, the ratios of Pakl and $\alpha$ Actin were calculated and plotted on a graph $(C)$. The results are from three independent experiments $(n=3)$.

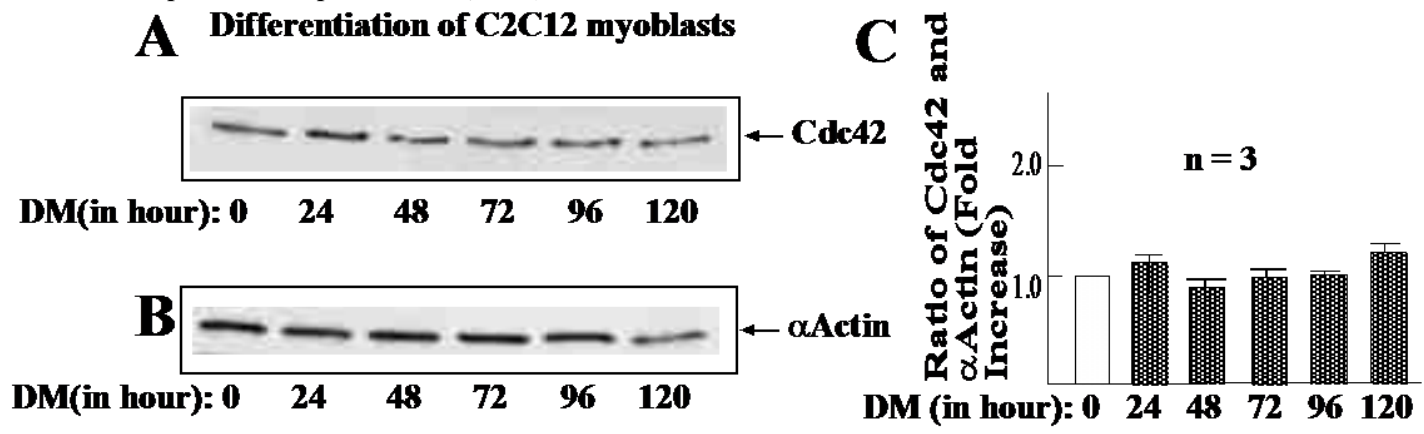

Fig4. Levels of Cdc42 in C2C12 mouse myoblasts undergoing differentiation into myotubes. Similar experiments as mentioned in legend to Figure 1 were performed except the total proteins samples were analyzed on Western blots for the levels of Cdc42 (A) and aActin (B) using specific antibodies to these proteins. After scanning the band intensities from panel $A$ and panel $B$, the ratios of $C d c 42$ and $\alpha A c t i n$ were calculated and plotted on a graph $(C)$. The results are from three independent experiments $(n=3)$.

\section{DiSCUSSION}

Skeletal muscle differentiation from myogenic cells takes place in various steps. Several proteins are involved in this most important cellular process. P67, which binds to both eIF2 and ERK1/2 kinases, is involved in skeletal muscle differentiation (13). C2C12 myoblasts withdraw permanently from cell cycle during differentiation into myotubes. While examining whether p67 has any roles in cytoskeleton dynamics we looked at the expression and activity of Pak1, a member of Pak family of proteins, which are involved in this process (31). Pak family of serine/threonine kinases are effectors for the Rho GTPases Cdc42 and Rac, which act as molecular switches controlling cytoskeletal remodeling, gene expression, cell proliferation, invasion, differentiation, and apoptosis (32). Major function of Paks is to control the cytoskeleton dynamics primarily through the regulation of polymerized actin structures, particularly the formation of filopodia and lamellipodia, consequently affecting the cell shape, motility, and adhesion (32). Group A Paks phosphorylates the regulatory myosin light chain VI, which is involved in membrane trafficking and cell migration (33). The ARP2/3 complex controls actin nucleation and branching. Phosphorylation of the p41-ARC subunit of ARP2/3 complex by Pak1 stimulates the assembly of the cellular cortex of migrating cells (32). Paks also play important roles in cell motility by regulating the leading edge microtubule dynamics (32). High levels of Pak in cells result in increased cell motility (34). The Pak1 knockout mice are deficit in 
glucose homeostasis, which includes inefficient insulin secretion and abnormal glucose clearance. This is reminiscent of the biological role of Pak1 in humans, where Pak1 levels are reduced in type 2 diabetic islets (35). These observations implicate Pakl's direct role in the control of glucose metabolism.

Although, the level of Pak1 did not change significantly during differentiation of C2C12 myoblasts in culture (Fig. 3), its level increases both in C2C12 myoblasts and myotubes constitutively expressing rat p67 (Figs. 1 and 2). P67 has growth inhibitory activity (14) and overexpression of p67 in C2C12 myoblasts possibly inhibits their growth prematurely that may lead to the differentiation even in growth medium. C2C12 myoblasts when shifted to differentiation medium, they differentiate into myotubes more efficiently and therefore the level of Pak1 is increased more significantly in myotubes constitutively expressing rat p67 (Fig. 2). Differentiation of C2C12 myoblasts is a slow process and series cellular events take place during this transition. Since the level of p67 gradually increases while C2C12 myoblasts were differentiating into myotubes and peaks at the time of myoblasts' fusion into myotubes (3-5), it is more likely that p67 increases Pak1 level transiently to induce cytoskeleton dynamics especially myoblasts' motility during formation of myotubes. Pak1's activity is regulated by its upstream effector Cdc42, which is activated by Rho GTPases (31). Although, the level of Cdc42 did change significantly while C2C12 myoblasts were differentiating into myotubes (Fig. 4), its level is increased in rat tumor hepatoma cells constitutively expressing rat p67 (Fig. 5). These results thus suggest that p67 is involved possibly transiently in the upregulated expression and proteolytic processing of Cdc42, which increases the activity of Pak1. Since high level of Pak1 is essential for cytoskeleton dynamics, especially survival, migration and motility of cells, it is thus conceivable that p67 is involved in these processes directly or indirectly by modulating the expression and activity of Pak1.
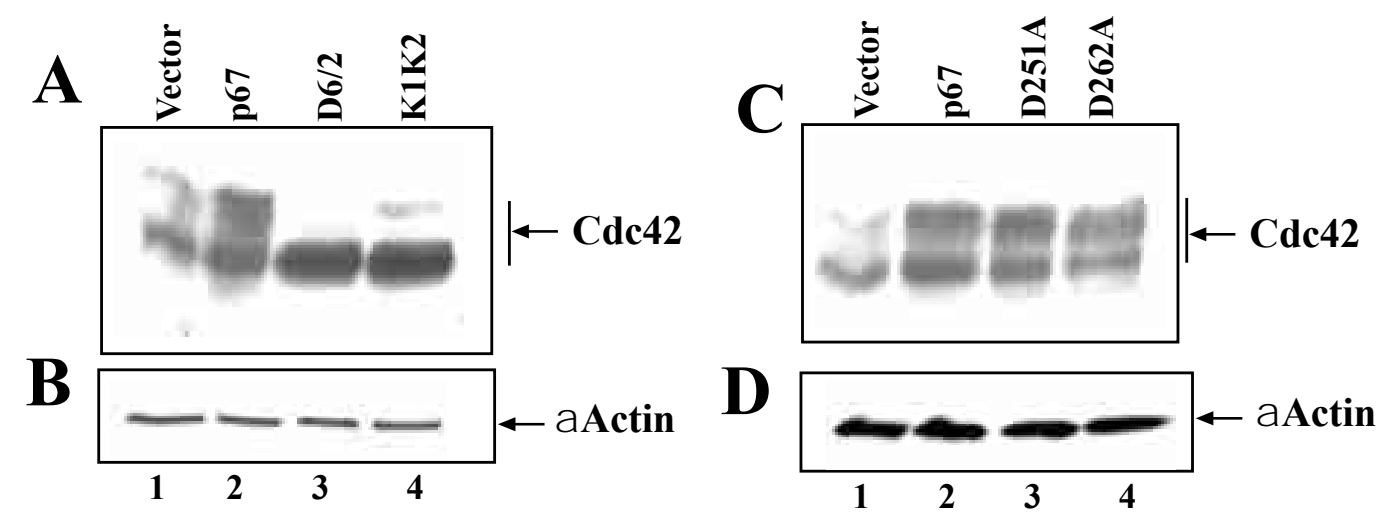

Fig5. Levels of Cdc42 in rat tumor hepatoma cells constitutively expressing EGFP vector and its in-frame fusions of rat p67 and its mutants D6/2, K1K2, D251A, and D262A. Stable cell lines constitutively expressing EGFP expresion vector, and its in-frame fusions of rat 067 or its various mutants were generated. Cell lysates from these cell lines were made and analyzed for the levels of $C d c 42(A \& C)$ and $\alpha A c t i n(B \& D)$ on Western blots using antibodies specific to these proteins. These experiments were repeated three times and a representitative result is shown.

\section{REFERENCES}

[1] Buckingham, M., and Rigby, P. W. J. (2014) Gene regulatory networks and transcriptional mechanisms that control myogenesis. Dev. Biol. 28, 225-238.

[2] Asp, P., Blum, R., Vethantham, V., Parisi, F., Micsinai, M., Cheng, J., Bowman, C., Kluger, Y., and Dynlacht, B. D. (2011) Genome-wide remodeling of the epigenetic landscape during myogenic differentiation. Proc. Natl. Acad. Sci. USA 108, E149-E158.

[3] Datta, B., Datta, R., Majumdar, A., and Ghosh, A. (2005) The stability of eukaryotic initiation factor 2-associated glycoprotein, p67, increases during skeletal muscle differentiation and that inhibits the phosphorylation of extracellular signal-regulated kinases 1 and 2. Exp. Cell Res. 303, 174-182.

[4] Datta, B., Datta, R., Majumdar, A., and Balusu, R. (2004) Treatment of cells with the angiogenic inhibitor, fumagillin results in increased stability of eukaryotic initiation factor 2-associated glycoprotein, p67, and reduced phosphorylation of extra-cellular signal-regulated kinases 1 and 2. Biochemistry 43, 14821-14831. 
[5] Datta, B. and Datta, R. (2014) Phosphorylation of eukaryotic initiation factor $2 \alpha$ during differentiation of mouse myoblasts into myotubes is mediated by an unknown kinase. Intl. J. Adv. Res. Chem. Sci. 1, 1-5.

[6] Knoblauch, A., Will, C., Goncharenko, G., Ludwig, S., and Wixler, V. (2007) The binding of Mss4 to $\alpha$-integrin subunits regulates matrix metalloproteinase activation and fibronectin remodeling. The FASEB J. 21, 498-510.

[7] Dedieu, S., Poussard, S., Mazeres, G., Grise, F., Dargelos, E., Cottin, P., and Brustis, J.-J. (2004) Myoblast migration is regulated by calpain through its involvement in cell attachment and cytoskeleton organization. Exp. Cell. Res. 292, 187-200.

[8] Datta, S., and Datta, B. (2016) Differential expression of MARCKS in C2C12 myoblasts and myotubes constitutively expressing p67. Intl. J. Adv. Res. Chem. Sci. 3(2), 22-29.

[9] Dedieu, S., Mazeres, G., Poussard, S., Brustis, J.-J., and Cottin, P. (2003) Myoblast migration is prevented by a calpain-dependent accumulation of MARCKS. Biol. Cell, 95, 615-623.

[10] Kumar, K. S., Ramadhas, A., Nayak, S. C., Kaniyappan, S., Dayma, K., and Radha, V. (2015) C3G (RapGEF1), a regulator of actin dynamics promotes survival and myogenic differentiation of mouse mesemchymal cells. Biochim. Biophys. Acta 1853, 2629-2639.

[11] Suetsugu, S. (2010) The proposed functions of membrane curvatures mediated by the BAR domain superfamily proteins. J. Biochem. 148, 1-12.

[12] Abmayr, S. M., and Pavlath, G. K. (2012) Myoblast fusion: lessons from flies and mice. Development 139, 641-656.

[13] Datta, B. (2015) Diversified roles of p67/MetAP2 as a regulator of cell growth and differentiation, in tumor suppression, and in obesity. Review, Curr. Topics Biochem. Res. 16, 41-52.

[14] Datta, B. (2009) Roles of P67/MetAP2 as a tumor suppressor - a review. Biochim. Biophys. Acta 1796, 281-292.

[15] Datta, B., Ray, M.K. Chakrabarti, D. Wylie, D. E and Gupta, N.K. (1989) Glycosylation of Eukaryotic Peptide Chain Initiation Factor 2 (eIF-2)-associated $67 \mathrm{kDa}$ Polypeptide $\left(\mathrm{p}^{67}\right)$ and Its Possible Role in the Inhibition of eIF-2 Kinase-catalyzed Phosphorylation of the eIF-2 $\alpha$-Subunit by eIF-2. J. Biol. Chem. 264, 20620-20624.

[16] Datta, R., Choudhury, P., Ghosh, A., and Datta, B. (2003) A glycosylation site, ${ }_{60} \mathrm{SGTS}_{63}$, of p67 is required for its ability to regulate the phosphorylation and activity of eukaryotic initiation factor2 alpha (eIF2 $\alpha$ ). Biochemistry 42, 5453-5460.

[17] Ghosh, A., Datta, R., Majumdar, A., Bhattacharya, M., and Datta, B. (2006) The N-terminal lysine residue-rich domain II and the 340-430 amino acid segment of eukaryotic initiation factor 2-associated glycoprotein p67 are the binding sites for the $\gamma$-subunit of eIF2. Exp. Cell Res. 312, 3184-3203.

[18] Majumdar, A., Ghosh, A., Datta, S., Prudner, B., and Datta, B. (2010) P67/MetAP2 suppresses K-RasV12 mediated transformation of NIH3T3 mouse fibroblasts in culture and in athymic mice. Biochemistry 49, 10146-10157.

[19] Datta, B., Ghosh, A., Majumdar, A., and Datta, R. (2007) Auto-proteolysis of rat p67 generates several peptide fragments: the N-terminal fragment, p26, is required for the protection of eIF2 $\alpha$ from phosphorylation. Biochemistry 46, 3465-3475.

[20] Liu, S., Widom, J., Kemp, C. W., Crews, C. M., and Clardy, J. (1998) Structure of human methionine aminopeptidase-2 complexed with fumagillin. Science 282, 1324-1327.

[21] Rane, C. K., and Minden, A. (2014) P21 activated kinases: structure, regulation, and functions. Small GTPases, 5, e28003.

[22] Manser, E., Leung, T., Salihuddin, H., Zhao, Z. S., and Lim, L. A. (1994) A brain serine/threonine protein kinase activated by Cdc42 and Rac1. Nature 367, 40-46.

[23] Whale, A., Hashim, F. N., Fram, S. Jones, G. E., Wells, C. M. (2011) Signalling to cancer cell invasion through PAK family of kinases. Front. Biosci. 16, 849-864. 
[24] Wu, S., Gupta, S., Chatterjee, N., Hileman, R.E., Kinzy, T.G., Denslow, N.D., Merrick, W.C., Chakrabarti, D., Osterman, J.C., and Gupta, N.K. (1993) Cloning and characterization of complementary DNA encoding the eukaryotic initiation factor 2-associated 67-kDa protein $\left(\mathrm{p}^{67}\right)$ J. Biol. Chem. 268, 10796-10801.

[25] Ghosh, A., Tammali, R., Balusu, R., Datta, R., Chattopadhyay, A., Bhattacharya, M., and Datta, B. (2014) Oligomerization of the eukaryotic initiation factor 2-associated glycoprotein p67 requires N-terminal 1-107 amino acid residues. Intl. J. Appl. Biotech. Biochem._4, 25-44.

[26] Datta, B., Datta, R., Ghosh, A., and Majumdar, A. (2004) Eukaryotic initiation factor 2associated glycoprotein, p67, shows differential effects on the activity of certain kinases during serum-starved conditions. Arch. Biochem. Biophys. 427, 68-78.

[27] Datta, B., Datta, R., Ghosh, A., and Majumdar, A. (2006) The binding between p67 and eukaryotic initiation factor 2 plays important roles in the protection of eIF $2 \alpha$ from phosphorylation by kinases. Arch. Biochem. Biophys. 452, 138-148.

[28] Ausubel, F. M., Brent R., Kingston R. E., Moore D. D., Seidman J. G., Smith J. A., Struhl K. (1992) Current Protocols in Molecular Biology, Vol. 1, section 8.1.

[29] Sambrook, J., Fritsch, E. F., and Maniatis, T. (1989) Molecular Cloning: A Laboratory manual, $2^{\text {nd }}$ edn., Cold Spring Harbor Laboratory Press, Cold spring Harbor, NY.

[30] Datta, B. Min, W., Burma, S., and Lengyel, P. (1998) Increase in p202 expression during skeletal muscle differentiation: Inhibition of MyoD protein expression and activity by $\mathrm{p} 202$. Mol. Cell. Biol. 18, 1074-1083.

[31] Dummler, B., Ohshiro, K., Kumar, R., Field, J. (2009) Pak protein kinases and their role in cancer. Cancer Metastasis Rev. 28, 51-63.

[32] Etienne-Manneville, S. (2004) Cdc42 - the centre of polarity. J. Cell. Sci. 117, 1291-1300.

[33] Buss, F., Kendrick-Jones, J., Lionne, C., Knight, A. E., Core, G. P., and Paul Luzio, J. (1998) The localization of myosin VI at the golgi complex and leading edge of fibroblasts and its phosphorylation and recruitment into membrane ruffles of A431 cell after growth factor stimulation. J. Cell. Biol. 143, 1535-1545.

[34] Ye, D. Z., and Field, J. (2012) PAK signaling in cancer. Cell Logist 2, 105-116.

[35] Wang, Z., Oh, E., Clapp, D. W., Chernoff, J., and Thurmond, D. C. (2011) Inhibition or ablation of p21-activating kinase (PAK1) disrupts glucose homeostatic mechanisms in vivo. J. Biol. Chem. 286, 41359-41367.

\section{AUTHOR'S BIOGRAPHY}

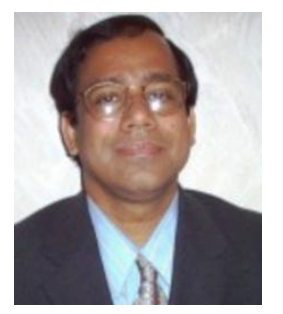

Bansidhar Datta, Ph.D., Associate Professor, Department of Chemistry \& Biochemistry, Kent State University, Kent, OH 44240, USA 